\title{
Evaluation of the Antimicrobial Activity of Leaf Extracts of Acokanthera schimperi against Various Disease-Causing Bacteria
}

\section{Ambaye Kenubih (D) \\ Eyerusalem Belay (D) \\ Kumneger Lemma}

University of Gondar, College of Veterinary Medicine and Animal Sciences, Gondar, Ethiopia
Correspondence: Ambaye Kenubih Email ambayken@yahoo.com
Background: In traditional medicine of Ethiopia, Acokanthera schimperi is the one used to treat different infectious diseases. Hence, this study was conducted with the main aim of determining active compounds in the leaves of $A$. schimperi.

Methods: The antimicrobial activities of using disk diffusion, minimum inhibitory concentration (MIC) and minimum bactericidal concentration (MBC) and acute oral toxicity of the fraction.

Results: The average bacterial zone of inhibition of the dichloromethane (DCM), chloroform (TCM), petroleum ether (PE) and ethyl acetate (EA) fractions ranged from $7.67 \mathrm{~mm}$ to $18.12 \mathrm{~mm}$. The average values of MIC of the DCM, TCM, PE and EA fractions ranged from $4.17 \mathrm{mg} / \mathrm{mL}$ to $33.34 \mathrm{mg} / \mathrm{mL}$. The most susceptible bacterium at $200 \mathrm{mg} / \mathrm{mL}$ was $S$. typhi, $(18.12 \mathrm{~mm})$, while the less susceptible bacterium was $C$. freundii $(14.33 \mathrm{~mm})$.

Conclusion: The solvent fractions demonstrated significant antibacterial activities with varying spectrum and safe up to $2000 \mathrm{mg} / \mathrm{kg}$.

Keywords: Acokanthera schipheri, acute toxicity, antibacterial activity, phytochemical profile and solvent fractions

\section{Introduction}

In Ethiopia, plant remedies are still the most important and sometimes the only source of therapeutics for nearly $80 \%$ of human and more than $90 \%$ of the livestock population. ${ }^{1}$ The country endowed with rich flora and fauna, due to its rugged topography and climatic diversity. For instance, Ethiopian flora is estimated to consist of about 7000 species. Out of these, it is believed that $10 \%$ are endemic to the country and about $14 \%$ are used as medicinal plants. ${ }^{2}$

One of the plants applicable in this study is Acokanthera schimperi (A.DC.) Schweinf, which grows in Ethiopia, and where it is known locally as Merz inchet (Amharic). It is a tree of dry woodland, thickets and grasslands 1100-2300 m, often at the margin of dry forest or forest remnants. It prefers rich, well-drained forest soil but also grows on black cotton and poor soils in the arid mid-lands. Acokanthera schimperi, has been reported to possess a varied range of therapeutical and pharmacological applications; traditionally it is used for wound, ${ }^{3-5}$ hepatitis, ${ }^{5,6}$ tonsillitis, ${ }^{7}$ malaria, ${ }^{8}$ scabies $^{9}$ and insect repellent. ${ }^{10}$

Phytochemical studies reveal that A. schimperi has a number of secondary metabolites; flavonoids, terpenes, alkaloids, coumarin, cardiac glycosides, 
anthraquinone, tannin and phenolic compounds. ${ }^{11,12}$ To the best of our search, there were no studies conducted beyond screening of the aqueous and hydroalcoholic extracts of the plant. ${ }^{13-15}$

Expertise in ethno-veterinary is important given that veterinary pharmaceuticals substantially reduce livestock mortality rates and by promoting prudent antibiotic use may preserve the long-term efficacy of drugs by limiting the development of antimicrobial resistance plant. ${ }^{16}$ Nonprudent drug usage is particularly concerning in lowincome countries where pharmaceutical use often occurs outside of a professional veterinary context plant. ${ }^{17}$ Nevertheless, it is believed that many more animals die due to a lack of access to antimicrobials compared to infections caused by resistance bacteria plant. ${ }^{17}$

Therefore, the present study was conducted to evaluate the antibacterial activity of different solvent fractions of Acokanthera schimperi leave on selected pathogenic bacterial species, to determine the phytochemical constituents of solvent fractions qualitatively and to determine the acute toxicity of solvent fractions of A. schimperi.

\section{Materials and Methods Study Area}

Leaves of A. schimperi were collected from their natural habitat around Arema natural forest, which is found in the Arema-biderkun peasant association, Takusa district $\left(12^{\circ}\right.$ $09^{\prime} 01^{\prime \prime} \mathrm{N}$ and $\left.39^{\circ} 12^{\prime} 01^{\prime \prime} \mathrm{E}\right)$, North Gondar, Ethiopia. The invitro experimental study was conducted in the microbiology laboratory of the College of Veterinary Medicine and Animal Sciences (CVMAS), University of Gondar, Ethiopia.

The experimental study involving an in-vitro antimicrobial activity of $A$. schimperi leave fractions on disease causing bacterial species and phytochemical screening were conducted in microbiology laboratory of College of Veterinary Medicine and Animal Sciences (CVMAS), University of Gondar from October 2018 to April 2019.

\section{Collection, Identification, and Preparation of Plant Materials}

The leave was identified at Addis Ababa University by an experienced botanist and the voucher specimen (AK0012) was deposited at the National Herbarium, College of Natural Science of Addis Ababa University, Ethiopia. The leaves were transported to the University of Gondar, College of Veterinary Medicine and Animal Science,
Veterinary Pharmacology Laboratory. Then, washed thoroughly with tap water and dried under shade. The dried leave materials were grinded by an electric mill.

\section{Drugs, Reagents and Bacteriological Media}

The solvents used for extracting the plant material were distilled water, methanol, dichloromethane dimethyl sulfoxide (DMSO), phosphate buffered saline, iodine, ferric chloride and Resazurin and all solvents $\left(40-60^{\circ} \mathrm{C}\right.$ bp) (LobaChemie, India). The standard antibiotic discs that were used in the antibacterial activity tests were purchased from Oxoid, UK. The bacteriological media that were used in the study include Mueller Hinton agar (MHA), 0.5\% McFarland standard, Resazurin, Mueller Hinton broth, Plate count agar (Tryptone glucose yeast extract agar) (HiMedia, India). All chemicals and reagents used were of laboratory grade.

\section{Plant Extraction and Fractionation}

Two kilo gram of $A$. schimperi leave powder were soaked in $10 \mathrm{~L}$ of $80 \%$ methanol and socked for $48 \mathrm{hr}$ in a flask, three times, and agitated thoroughly to mix the slurry. The extracts were filtered using Whatman filter paper No. 1 and the aqueous crude extracts were kept at $4^{\circ} \mathrm{C}$ and all the three extracts were mixed and subjected to evaporation by a Rota-vaporizer (Buchi, England) which was set at $50^{\circ} \mathrm{C}$ and then after it were dried by using a freeze drier (Ningbo, China).

Fractionation was done according to ${ }^{18}$ with slight modification of the steps. Briefly, 200g of crude methanol extract was suspended in $100 \mathrm{~mL}$ distilled water and transferred into a separation funnel. Thirty milliliters of n-hexane, dichloromethane and petroleum ether were added separately to it and shaken by hand for some minutes. Then the mixture was left to have a separate mixture and the upper layer or the lower layers were separated depending on the solvent. The solvent fractions were taken (dichloromethane, chloroform, petroleum ether and ethyl acetate) and evaporated using a Rota-vaporizer to obtain the fraction.

\section{Phytochemical Screening}

The dichloromethane, chloroform, ethyl acetate and petroleum ether fractions of $A$. schimperi leave were screened for the presence phenolic compounds, flavonoids, terpenoids, cardiac glycosides, alkaloids, saponins and tannins 
were performed using standard phytochemical procedures as stated elsewhere ${ }^{19}$ and. $^{20}$

\section{Antimicrobial Assay}

To screen the antibacterial effect of the fractions using the disk diffusion method according to, ${ }^{21}$ with slight modification. Briefly, 6mm (diameter) discs were prepared from Whatman No. 1 filter paper. The discs were sterilized by autoclaving. Then, disks were soaked in $50 \mu \mathrm{L}$ fractions with a concentration of $200 \mathrm{mg} / \mathrm{mL}, 100 \mathrm{mg} / \mathrm{mL}, 50 \mathrm{mg} / \mathrm{mL}$ and $25 \mathrm{mg} / \mathrm{mL}$ of the solvent fractions. ${ }^{22,23}$

The microbial suspensions were prepared by making a saline suspension of isolated colonies selected from nutrient agar, and the agar plates were grown for 18-24 $\mathrm{h}$. The suspension was adjusted to match the tube of 0.5 McFarland turbidity standard, which equals to $1 \times 10^{8} \mathrm{CFU} / \mathrm{mL}$.

A $6 \mathrm{~mm}$ diameter sterilized Whatman filter paper discs were impregnated with $100 \mu \mathrm{L}$ of $200 \mathrm{mg} / \mathrm{mL}, 100 \mathrm{mg} / \mathrm{mL}$, $50 \mathrm{mg} / \mathrm{mL}$ and $25 \mathrm{mg} / \mathrm{mL}$ of each of the solvent fractions. Standard antimicrobial discs (vancomycin and chloramphenicol) were used as a positive control and $100 \mu \mathrm{L}$ of $5 \%$ dimethyl sulfoxide (DMSO) was used as a negative control. Then incubation of the media at $37^{\circ} \mathrm{C}$ for $24 \mathrm{hrs}$ in an inverted position. Then, it was incubated at $37^{\circ} \mathrm{C}$ for 24 hours. The zone of inhibition was measured using digital caliper. The experiment was performed in three independent tests for each bacterial strain and the mean of zones of inhibition was calculated for each fraction.

The minimum inhibitory concentration (MIC) was conducted according to CLSI. ${ }^{24}$ Briefly $100 \mu \mathrm{L}$ Muller Hinton Broth (MHB) was transferred aseptically to the well of a 96-well micro-titer plate. The stock solutions of the fractions were diluted in $5 \% \mathrm{DMSO}$, and then $100 \mu \mathrm{L}$ of stock solution containing approximately $200 \mathrm{mg} / \mathrm{mL}$ of each fraction was transferred aseptically to the first six rows and serial two-fold dilution to the 10th column by transferring $100 \mu \mathrm{L}$ sequentially. The bacterial strains were prepared using MHB then $0.1 \mathrm{~mL}$ was diluted with $1 \mathrm{~mL}$ of distilled water in the ratio of 1:1000 to give a final dilution of $10^{5} \mathrm{CFU} / \mathrm{mL}$ of the standard inoculums following the dilution susceptibility technique. Fifty microliter of the adjusted inoculum (turbidity adjusted equivalent to 0.5 McFarland) was transferred aseptically to the 96-well micro-titer plate up to 10 th of the wells containing the test dilutions, negative control (11th column) and sterility control (12th column). Then, $30 \mu \mathrm{L}$ resazurin was added as an indicator for bacterial growth; bacteria metabolize it and change its color to pink. ${ }^{25,26}$ The micro-titer plates were incubated at $37^{\circ} \mathrm{C}$ for $24 \mathrm{hrs}$ for all the organisms. The wells which had no change in color after the $24 \mathrm{hrs}$ incubation indicated no growth of the microorganisms and they were taken as MIC values. The tests were run in triplicate.

The minimum bactericidal concentration (MBC) is defined as the lowest concentration where no bacterial growth is observed. It was determined by first selecting tubes that showed no growth during MIC determination; $3 \mu \mathrm{L}$ samples were taken and streaked on the surface of plate count agar. The plates were allowed to dry and then kept in an inverted position in an incubator at $37^{\circ} \mathrm{C}$ for 24 hrs. After the incubation period, the colonies were counted and compared with the negative control. The concentration of the plant extract that completely inhibited the growth of the test organism was considered as MBC. ${ }^{25,26}$

\section{Acute Toxicity}

Acute toxicity test $\left(\mathrm{LD}_{50}\right)$ was conducted by using female white albino mice which were brought from the Ethiopian Public Health Institute (EPHI); Addis Ababa, Ethiopia. These experiment animals weigh 25-30g (average weight) and are 9 weeks of age. Prior to using the experiments, it starved for $3 \mathrm{hrs}$. It was kept in plastic cages at $22^{\circ} \mathrm{C}$ and on a $12 \mathrm{hr}$ light dark cycle with free access to pellet food and water. ${ }^{27}$ This study was done using the limit test dose of $2000 \mathrm{mg} / \mathrm{kg}$ according to the Organization for Economic Cooperation and Development (OECD) guideline for testing of chemicals using white albino mice. ${ }^{28}$ They were randomly distributed into five groups. The first group (control group) received orally distilled water $(10 \mathrm{~m} 1 / \mathrm{kg})$ as negative control. The white albino mice groups $(n=3)$ with the same sex and age were individually given orally $2000 \mathrm{mg} / \mathrm{kg}$ of DCM, TCM, PE and EA fraction of A. schimperi.

\section{Ethical Approval}

All animals were maintained on a standard mouse diet and water was provided ad-libitum. All animal experimentation was done in accordance with the University of Gondar, Research and community service vice president office. Ethical clearance with approval number O/V/P/RC/ 05/813 was obtained from the Research and community service vice president office, University of Gondar before the commencement of the study. All animal experiments were conducted in accordance with the National Institutes 
of Health guide for the care and use of laboratory animals (NIH Publications No. 8023, revised 1978).

\section{Statistical Analysis}

The experimental data is expressed as mean \pm Standard Error of the Mean (SEM). Data are analyzed using the Statistical Package for the Social Sciences (SPSS), version 24.0 software. The statistical differences in the mean zone of inhibition of solvent fractions for individual bacterium were carried out by ANOVA followed by a Tukey Post Hoc Multiple Comparison test at a significance level of $\mathrm{P}<0.05$. The MIC and MBC values of fractions were carried out by one way analysis of variance. Moreover, the concentration dependent antibacterial activities of the dichloromethane and petroleum fractions for each bacterium were determined by independent $t$-test analysis using the SPSS software.

\section{Results}

\section{Phytochemical Screening}

The result of the Phytochemical screening test is shown in Table 1. According to the qualitative Phytochemical screening of the DCM, TCM, EA, and PE fractionates of the leaf of $A$. schimperi were found to be positive for the presence of all of the tested secondary metabolites.

\section{Antibacterial Activity}

According to the agar well diffusion test, the growth of all test bacterial strains was inhibited by the tested concentrations of DCM, TCM, PE, and EA fraction of A. schimperi. However, there were a statistically significant difference as compared to that of their respective positive control $(\mathrm{p}<0.05)$ against all test bacteria (Tables $2-5)$.

E. coli, S. aureus, K. pneumoniae by PE fraction were among the most susceptible than those of the rest of the tested bacterial species. As indicated in (Table 2), the most susceptible bacteria at $200 \mathrm{mg} / \mathrm{mL}$ were E. coli, S. typhi and
K. pneumonia on DCM fraction with the same mean zone of inhibition of $16.67 \mathrm{~mm}$ while E. coli, S. aureus and P. aeruginosa with the mean zone of inhibition of $17.67 \mathrm{~mm}$, $17.33 \mathrm{~mm}$ and $17.33 \mathrm{~mm}$ by PE fractionate, respectively. S. typhi showed a higher zone of inhibition in the EA and in the TCM fractions, $18.25 \mathrm{~mm}$ and $18.00 \mathrm{~mm}$, respectively.

With the exception of $C$. freundii which appears relatively resistant to four fractionates. The zone of inhibition of the PE fraction was greater than that of the DCM fraction at equal concentrations against the growth of each test bacterium except $S$. typhi, with a significant difference $(\mathrm{p}<0.05)$. The TCM fraction (Figure $1 \mathrm{~A})$ and EA fractions (Figure 1B) exhibits a low inhibition zone against S. typhi; while the PE fraction (Figure 2) exhibit low inhibition zone against $K$. pneumonia.

\section{Minimum Inhibitory Concentration of Solvent Fractions}

The MIC value of the DCM fraction ranges from $4.17 \mathrm{mg} /$ $\mathrm{mL}$ to $22.24 \mathrm{mg} / \mathrm{mL}$ in all bacterial species for which it was active, with the lowest MIC (against $K$. pneumoniae) and maximum MIC (against $C$. freundii) respectively. The highest MIC value of PE fraction was $33.34 \mathrm{mg} / \mathrm{mL}$ (against $C$. freundii) and the lowest MIC value was $11.13 \mathrm{mg} / \mathrm{mL}$ (against K. pneumoniae, P. aeruginosa, and $S$. typhi) with equal values. When compared the MIC value of solvent fractions; DCM fraction was more effective against all tested bacteria (Table 6).

The MIC value of the TCM fraction ranged from $8.35 \mathrm{mg} / \mathrm{mL}$ to $55.56 \mathrm{mg} / \mathrm{mL}$ in all bacterial species for which it was active with lowest MIC (against $S$. typhi) and maximum MIC (against $C$. freundii) respectively. The highest MIC value of EA fraction was $44.45 \mathrm{mg} / \mathrm{mL}$ (against $C$. freundii) and the lowest MIC value was $4.17 \mathrm{mg} / \mathrm{mL}$ (against K. pneumoniae, P. aeruginosa, and S. typhi) with equal values (Table 7 ).

Table I Phytochemical Investigation of Solvent Fractions of A. schimperi Leave

\begin{tabular}{|l|l|l|l|l|}
\hline Phytochemical Tests & DCM Fractionate & TCM Fraction & PE Fractionate & EA Fraction \\
\hline Terpenoids & + & + & + & \\
Tannin & + & + & + & + \\
Alkaloid & + & + & + & + \\
Cardiac glycoside & - & - & + & - \\
Phenolic compounds & + & + & + & + \\
Saponin & + & + & + & + \\
Flavonoids & + & + & + & + \\
\hline
\end{tabular}

Abbreviations: +, present; -, absent. 
Table 2 Zone of Inhibition (in $\mathrm{mm}$ ) of the Different Concentrations of DCM Fraction of A. schimperi Leave Against the Tested Bacteria

\begin{tabular}{|c|c|c|c|c|c|c|}
\hline & \multicolumn{6}{|l|}{ DCM } \\
\hline & $200 \mathrm{mg} / \mathrm{mL}$ & $100 \mathrm{mg} / \mathrm{mL}$ & $50 \mathrm{mg} / \mathrm{mL}$ & $25 \mathrm{mg} / \mathrm{mL}$ & Vanc & CAF \\
\hline C. freundii(ATCC 43864) & $13.33 \pm 0.66^{\mathrm{a}^{* * * *} \mathrm{~b} * * * c^{*}}$ & $11.67 \pm 0.88^{\mathrm{a}^{* * * *} \mathrm{~b} * * *}$ & $9.67 \pm 0.88^{\mathrm{a}^{* * * *} \mathrm{e}^{*}}$ & $7.67 \pm 0.33^{\mathrm{a}^{* * * *} \mathrm{~d} * * *} \mathrm{e}^{* * * *}$ & $\begin{array}{l}22.33 \\
\pm 0.33\end{array}$ & $\begin{array}{l}22.67 \\
\pm 0.33\end{array}$ \\
\hline $\begin{array}{l}\text { K. pneumonia (ATCC } \\
700603 \text { ) }\end{array}$ & $16.67 \pm 0.66^{\mathrm{a} * * * \mathrm{~b} * * * \mathrm{c} * *}$ & $14.33 \pm 0.88^{\mathrm{a}^{* * * *} \mathrm{~b}^{* * * *} \mathrm{c}^{*}}$ & II $.00 \pm 0.57^{\mathrm{a} * * * \mathrm{~d} * \mathrm{e} * * *}$ & $8.67 \pm 0.33^{\mathrm{a}^{* * * *} \mathrm{~d} * * * *} \mathrm{e}^{* * * *}$ & $\begin{array}{l}24.00 \\
\pm 0.57\end{array}$ & $\begin{array}{l}21.67 \\
\pm 0.66\end{array}$ \\
\hline S. typhi (ATCC 6539) & $\begin{array}{l}16.67 \\
\pm 0.33^{a^{* * * *} b * * * * * * * d^{*}}\end{array}$ & $\begin{array}{l}14.00 \\
\pm 0.33^{\mathrm{a}^{* * * *} \mathrm{~b} * * * *} \mathrm{c}^{*} \mathrm{e}^{*}\end{array}$ & $\begin{array}{l}11.67 \\
\pm 0.33^{3^{* * * * b * *} d^{*} e^{* * * *}}\end{array}$ & $\begin{array}{l}9.67 \\
\pm 0.33^{\mathrm{a}^{* * * *} \mathrm{c} * \mathrm{~d} * * * *} \mathrm{e}^{* * *}\end{array}$ & $\begin{array}{l}22.67 \\
\pm 0.66\end{array}$ & $\begin{array}{l}22.33 \\
\pm 0.33\end{array}$ \\
\hline E. coli (ATCC 25922)) & $\begin{array}{l}16.67 \\
\pm 0.33^{a^{* * * *} b^{* * * * * * * * d^{*}}}\end{array}$ & $\begin{array}{l}15.00 \\
\pm 0.00^{\mathrm{a}^{* * *} \mathrm{~b} * * * c^{* * *} \mathrm{e}^{*}}\end{array}$ & $\begin{array}{l}13.00 \\
\pm 0.00^{\mathrm{a}^{* * * *} \mathrm{~b}^{*} \mathrm{~d}^{* * *} \mathrm{e} * * *}\end{array}$ & $\begin{array}{l}I I .33 \\
\pm 0.33^{\mathrm{a}^{* * * *} \mathrm{c}^{*} \mathrm{~d}^{* * * *} \mathrm{e}^{* * * *}}\end{array}$ & $\begin{array}{l}24.00 \\
\pm 0.57\end{array}$ & $\begin{array}{l}23.33 \\
\pm 0.33\end{array}$ \\
\hline P. aeruginosa(ATCC) & $\begin{array}{l}15.67 \\
\pm 0.33^{\mathrm{a} * * \mathrm{~b}^{* * * *} \mathrm{c} * * *}\end{array}$ & 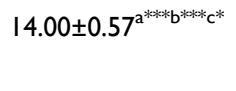 & $1 \mathrm{I} .67 \pm 0.33^{\mathrm{a} * * * \mathrm{~d} * \mathrm{e}^{* * * *}}$ & $9.67 \pm 0.33^{\mathrm{a} * * * \mathrm{~d} * * * \mathrm{e} * * *}$ & $\begin{array}{l}22.33 \\
\pm 0.66\end{array}$ & $\begin{array}{l}22.33 \\
\pm 0.66\end{array}$ \\
\hline S. aureus(ATCC 292I3) & $\begin{array}{l}15.00 \\
\pm 0.57^{\mathrm{a}^{* * *} \mathrm{~b} * * * * * * * *}\end{array}$ & $\begin{array}{l}13.33 \\
\pm 0.33^{\mathrm{a}^{* * * *} \mathrm{~b} * * * *} \mathrm{c}^{* *}\end{array}$ & $\begin{array}{l}10.67 \\
\pm 0.33^{\mathrm{a}^{* * *} \mathrm{~b} * \mathrm{~b}^{*} \mathrm{e} * * *}\end{array}$ & $\begin{array}{l}8.67 \\
\pm 0.33^{\mathrm{a}^{* * * *} \mathrm{c}^{*} \mathrm{~d}^{* * * *} \mathrm{e}^{* * * *}}\end{array}$ & $\begin{array}{l}19.33 \\
\pm 0.33\end{array}$ & $\begin{array}{l}22.33 \\
\pm 0.33\end{array}$ \\
\hline
\end{tabular}

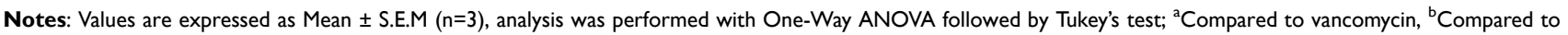
$25 \mathrm{mg} / \mathrm{mL}$, ${ }^{\mathrm{C}} \mathrm{To} 50 \mathrm{mg} / \mathrm{mL},{ }^{\mathrm{d}} \mathrm{To} 100 \mathrm{mg} / \mathrm{mL}$, ${ }^{\mathrm{e}} \mathrm{To} 200 \mathrm{mg} / \mathrm{mL} ;{ }^{*} \mathrm{P}<0.05, * * \mathrm{P}<0.0 \mathrm{I},{ }^{*} * * \mathrm{P}<0.00 \mathrm{I}$. The negative control has shown no antibacterial activity.

Abbreviations: CAF, chloramphenicol; Vacho, vancomycin.

\section{Minimum Bactericidal Concentration of Solvent Fractions}

The maximum mean MBC (least dilution) was $33.34 \mathrm{mg} / \mathrm{mL}$ (against $C$. freundii) by $\mathrm{PE}$ fractionate and the minimum mean MBC (highest dilution) of the study plant was $11.13 \mathrm{mg} / \mathrm{mL}$ (against $K$. pneumoniae, S. typhi and
P. aeruginosa). The corresponding values of the DCM fraction range from $6.95 \mathrm{mg} / \mathrm{mL}$ to $22.24 \mathrm{mg} / \mathrm{mL}$ for $K$. pneumoniae and C. freundii, respectively. Generally, the DCM fractionate was more potent in stopping bacterial growth against all tested bacteria's with a lower concentration compared to that of the PE fractionate (Tables 6 and 7).

Table 3 Zone of Inhibition (in mm) of the Different Concentrations of TCM Fraction of A. schimperi Leave Against the Tested Bacteria

\begin{tabular}{|c|c|c|c|c|c|c|}
\hline & \multicolumn{6}{|l|}{ TCM } \\
\hline & $200 \mathrm{mg} / \mathrm{mL}$ & $100 \mathrm{mg} / \mathrm{mL}$ & $50 \mathrm{mg} / \mathrm{mL}$ & $25 \mathrm{mg} / \mathrm{mL}$ & Vanc & CAF \\
\hline C. freundii(ATCC 43864) & $\begin{array}{l}14.33 \\
\pm 0.333^{\mathrm{a}^{* * * *} \mathrm{~b} * * * * * * *} \mathrm{~d}^{* *}\end{array}$ & $\begin{array}{l}11.67 \\
\pm 0.333^{\mathrm{a}^{* * * *} \mathrm{~b}^{* * * * *} \mathrm{c}^{* * * *} \mathrm{e}^{* *}}\end{array}$ & $8.33 \pm 0.333^{\mathrm{a}^{* * * * *} \mathrm{~d}^{* * * *} \mathrm{e}^{* * * *}}$ & $\begin{array}{l}6.83 \\
\pm 0.167^{\mathrm{a}^{* * * *} \mathrm{~d} * * *} \mathrm{e}^{* * *}\end{array}$ & $\begin{array}{l}22.33 \\
\pm 0.33\end{array}$ & $\begin{array}{l}22.67 \\
\pm 0.33\end{array}$ \\
\hline $\begin{array}{l}\text { K. pneumonia (ATCC } \\
700603 \text { ) }\end{array}$ & $\begin{array}{l}15.67 \\
\pm 0.667^{\mathrm{a}^{* * * *} \mathrm{~b} * * * c^{* * * *}}\end{array}$ & $\begin{array}{l}14.00 \\
\pm 0.577^{\mathrm{a}^{* * * *} \mathrm{~b} * * * \mathrm{c} * * *}\end{array}$ & $9.67 \pm 0.333^{\mathrm{a}^{* * * *} \mathrm{~d} * * * * * * * *}$ & $\begin{array}{l}8.17 \\
\pm 0.167^{\mathrm{a}^{* * * *} \mathrm{~d} * * *} \mathrm{e}^{* * *}\end{array}$ & $\begin{array}{l}24.00 \\
\pm 0.57\end{array}$ & $\begin{array}{l}21.67 \\
\pm 0.66\end{array}$ \\
\hline S. typhi (ATCC 6539) & $\begin{array}{l}18.0 \\
\pm 0.00^{a^{* * *} b^{* * * *} c^{* * *} d^{* * *}}\end{array}$ & $\begin{array}{l}15.33 \\
\pm 0.333^{\mathrm{a}^{* * *} \mathrm{~b} * * * \mathrm{c}^{*} * * * *} \mathrm{e}^{* * * *}\end{array}$ & $\begin{array}{l}10.33 \\
\pm 0.333^{\mathrm{a}^{* * *} \mathrm{~b} * * * \cdot \mathrm{d} * * *} \mathrm{e} * * *\end{array}$ & $\begin{array}{l}8.17 \\
\pm 0.167^{\mathrm{a}^{* * *} \mathrm{c} * * *} \mathrm{~d} * * * \mathrm{e} * * *\end{array}$ & $\begin{array}{l}22.67 \\
\pm 0.66\end{array}$ & $\begin{array}{l}22.33 \\
\pm 0.33\end{array}$ \\
\hline E. coli (ATCC 25922)) & $\begin{array}{l}16.25 \\
\pm 0.333^{\mathrm{a}^{* * * *} \mathrm{~b} * * * *} \mathrm{c} * * * \mathrm{~d} * *\end{array}$ & $14 \pm 0.00^{\mathrm{a}^{* * * *} \mathrm{~b} * * * \mathrm{c}^{* * *} \mathrm{e}^{* * *}}$ & 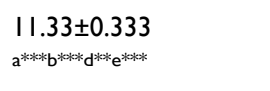 & $\begin{array}{l}7.5 \\
\pm 0.289^{\mathrm{a} * * *} \mathrm{c} * * * \mathrm{~d} * * * \mathrm{e} * * *\end{array}$ & $\begin{array}{l}24.00 \\
\pm 0.57\end{array}$ & $\begin{array}{l}23.33 \\
\pm 0.33\end{array}$ \\
\hline P. aeruginosa & $\begin{array}{l}17.67 \\
\pm 0.333^{\mathrm{a}^{* * * *} \mathrm{~b} * * * * * * * *} \mathrm{~d}^{* *}\end{array}$ & $\begin{array}{l}15.33 \pm \\
0.333^{\mathrm{a}^{* * *} \mathrm{~b} * * * *} \mathrm{c}^{* * * *} \mathrm{e}^{* *}\end{array}$ & $12 \pm 0.00^{\mathrm{a}^{* * * *} \mathrm{~d}^{* * * *} \mathrm{e}^{* * * *}}$ & 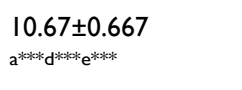 & $\begin{array}{l}22.33 \\
\pm 0.66\end{array}$ & $\begin{array}{l}22.33 \\
\pm 0.66\end{array}$ \\
\hline S. aureus(ATCC 29213) & $\begin{array}{l}16.25 \\
\pm 0.577^{\mathrm{a}^{* * * *} \mathrm{~b} * * * c_{\mathrm{c}}^{* * * *} \mathrm{~d} * *}\end{array}$ & $15 \pm 0.00^{\mathrm{a}^{* * * *} \mathrm{~b} * * * *} \mathrm{c}^{* * * *} \mathrm{e}^{* * * *}$ & $\begin{array}{l}10.33 \pm 0.333 \\
\mathrm{a}^{* * *} \mathrm{~b} * * * \mathrm{~d}^{* * *} \mathrm{e}^{* * * *}\end{array}$ & $\begin{array}{l}7 \\
\pm 0.577^{\mathrm{a}^{* * * *} c^{* * * *} \mathrm{~d} * * *} \mathrm{e}^{* * * *}\end{array}$ & $\begin{array}{l}18.33 \\
\pm 0.33\end{array}$ & $\begin{array}{l}22.33 \\
\pm 0.33\end{array}$ \\
\hline
\end{tabular}

Notes: Values are expressed as Mean \pm S.E.M $(n=3)$, analysis was performed with One-Way ANOVA followed by Tukey's test; ${ }^{a}$ Compared to vancomycin, ${ }^{b}$ Compared to $25 \mathrm{mg} / \mathrm{mL}$, 'To $50 \mathrm{mg} / \mathrm{mL}$, ${ }^{d}$ To $100 \mathrm{mg} / \mathrm{mL}$, ${ }^{\text {To }} 200 \mathrm{mg} / \mathrm{mL}$; $* * \mathrm{P}<0.0 \mathrm{I}$, $* * * \mathrm{P}<0.00 \mathrm{I}$. The negative control has shown no antibacterial activity. Abbreviations: CAF, chloramphenicol; Vacho, vancomycin. 
Table 4 Zone of Inhibition (in $\mathrm{mm}$ ) of the Different Concentrations of PE Fraction of A. schimperi Leave Against the Tested Bacteria

\begin{tabular}{|c|c|c|c|c|c|c|}
\hline & \multicolumn{6}{|l|}{ PE } \\
\hline & $200 \mathrm{mg} / \mathrm{mL}$ & $100 \mathrm{mg} / \mathrm{mL}$ & $50 \mathrm{mg} / \mathrm{mL}$ & $25 \mathrm{mg} / \mathrm{mL}$ & Vanc & CAF \\
\hline C. freundii(ATCC 43864) & $\begin{array}{l}13.67 \\
\pm 0.33^{\mathrm{a}^{* * * *} \mathrm{~b} * * * *} \mathrm{c}^{*} \mathrm{e}^{* *}\end{array}$ & $\begin{array}{l}11.67 \\
\pm 0.33^{3^{* * * *} b^{* * * *} c^{*} e^{* * *}}\end{array}$ & $\begin{array}{l}10.00 \\
\pm 0.00^{\mathrm{a}^{* * * *} \mathrm{~b} * \mathrm{~d}^{*} \mathrm{e} * * * *}\end{array}$ & $\begin{array}{l}8.33 \\
\pm 0.33^{\mathrm{a}^{* * * *} c^{*} \mathrm{~d}^{* * * *} \mathrm{e}^{* * * *}}\end{array}$ & $\begin{array}{l}22.33 \\
\pm 0.33\end{array}$ & $\begin{array}{l}22.67 \\
\pm 0.33\end{array}$ \\
\hline $\begin{array}{l}\text { K. pneumonia (ATCC } \\
700603 \text { ) }\end{array}$ & $\begin{array}{l}17.00 \\
\pm 0.00^{\mathrm{a}^{* * * *} \mathrm{~b} * * * \mathrm{c}} \mathrm{c}^{* * * * \mathrm{~d} * *}\end{array}$ & $\begin{array}{l}14.67 \\
\pm 0.33^{\mathrm{a}^{* * * *} \mathrm{~b} * * * c^{* * *} \mathrm{e} * *}\end{array}$ & $\begin{array}{l}12.00 \\
\pm 0.00^{\mathrm{a}^{\mathrm{a} * * \mathrm{~b}} \mathrm{~b} \cdot \mathrm{d} * * \mathrm{*} * * *}\end{array}$ & $\begin{array}{l}10.33 \\
\pm 0.33^{\mathrm{a}^{* * * * *} \mathrm{c}^{* * *+\mathrm{d}^{* * * *} \mathrm{e} * * * *}}\end{array}$ & $\begin{array}{l}24.00 \\
\pm 0.57\end{array}$ & $\begin{array}{l}21.67 \\
\pm 0.66\end{array}$ \\
\hline S. typhi (ATCC 6539) & $\begin{array}{l}16.00 \\
\pm 0.00^{\mathrm{a}^{* * * *} \mathrm{~b} * * * *} \mathrm{c}^{* * * *} \mathrm{~d}^{* *}\end{array}$ & $\begin{array}{l}14.00 \\
\pm 0.00^{\mathrm{a}^{* * *} \mathrm{~b} * * * *} \mathrm{c}^{* * *} \mathrm{*} * *\end{array}$ & $\begin{array}{l}11.67 \\
\pm 0.33^{\mathrm{a}^{* * *} \mathrm{~b} * *} \mathrm{d***} \mathrm{e}^{* * * *}\end{array}$ & $\begin{array}{l}9.67 \\
\pm 0.33^{a^{* * * *} c^{* * *} d^{* * * *} e^{* * * *}}\end{array}$ & $\begin{array}{l}22.67 \\
\pm 0.66\end{array}$ & $\begin{array}{l}22.33 \\
\pm 0.33\end{array}$ \\
\hline E. coli (ATCC 25922)) & $\begin{array}{l}17.67 \\
\pm 0.33^{\mathrm{a}^{* * *} \mathrm{~b} * * * *} \mathrm{c}^{* * * *} \mathrm{~d}^{* *}\end{array}$ & $15.33 \pm 0.33^{a * * * b^{* * * * *} \mathrm{e}^{* * *}}$ & $13.67 \pm 0.33^{* * * * \mathrm{~b} * \mathrm{e}^{* * * *}}$ & $\begin{array}{l}11.00 \\
\pm 0.57^{\mathrm{a}^{* * * *} \mathrm{c}^{* * *} \mathrm{~d} * * * *} \mathrm{e}^{* * * *}\end{array}$ & $\begin{array}{l}24.00 \\
\pm 0.57\end{array}$ & $\begin{array}{l}23.33 \\
\pm 0.33\end{array}$ \\
\hline P. aeruginosa & $\begin{array}{l}17.33 \pm \\
0.33^{\mathrm{a}^{* * * *} \mathrm{~b} * * * c^{*} * * * \mathrm{~d}^{* * *}}\end{array}$ & $\begin{array}{l}14.67 \\
\pm 0.33^{a^{* * * * b} \mathrm{~b} * * * * * * * * *} \mathrm{c}^{* *}\end{array}$ & $\begin{array}{l}12.00 \\
\pm 0.57^{a^{* * * * b} \mathrm{~b} * \mathrm{~d}^{* * *} \mathrm{e}^{* * * * *}}\end{array}$ & $\begin{array}{l}9.33 \\
\pm 0.33^{\mathrm{a}^{* * * * * * * * d * * * * * * * * *}}\end{array}$ & $\begin{array}{l}22.33 \\
\pm 0.66\end{array}$ & $\begin{array}{l}22.33 \\
\pm 0.66\end{array}$ \\
\hline S. aureus(ATCC 292I3) & $\begin{array}{l}17.33 \\
\pm 0.33^{\mathrm{a}^{* * *} \mathrm{~b} * * * *} \mathrm{c}^{* * * *} \mathrm{~d} * *\end{array}$ & $\begin{array}{l}15.00 \\
\pm 0.00^{\mathrm{a}^{* * * *} \mathrm{~b} * * * *} \mathrm{c}^{* * *} \mathrm{e}^{* * *}\end{array}$ & $\begin{array}{l}12.00 \\
\pm 0.57^{\mathrm{a}^{* * * * \mathrm{~b}} \mathrm{b*} \mathrm{d}^{* * *} \mathrm{e}^{* * * *}}\end{array}$ & $\begin{array}{l}9.67 \\
\pm 0.33^{\mathrm{a}^{* * * *} \mathrm{c}^{* * *} \mathrm{~d}^{* * * *} \mathrm{e}^{* * * *}}\end{array}$ & $\begin{array}{l}18.33 \\
\pm 0.33\end{array}$ & $\begin{array}{r}22.33 \\
\pm 0.33\end{array}$ \\
\hline
\end{tabular}

Notes: Values are expressed as Mean \pm S.E.M $(n=3)$, analysis was performed with One-Way ANOVA followed by Tukey's test; ${ }^{a}$ Compared to positive control, ${ }^{\text {b}} \mathrm{To} 25 \mathrm{mg} /$ $\mathrm{mL}$, ${ }^{\mathrm{C}} \mathrm{To} 50 \mathrm{mg} / \mathrm{mL}$, ${ }^{\mathrm{d}} \mathrm{To} 100 \mathrm{mg} / \mathrm{mL}$, ${ }^{\mathrm{e}} \mathrm{To} 200 \mathrm{mg} / \mathrm{mL} ;{ }^{*} \mathrm{P}<0.05$, $* * \mathrm{P}<0.01$, $* * * \mathrm{P}<0.001$. The negative control has shown no antibacterial activity.

Abbreviations: CAF, chloramphenicol; Vacho, vancomycin.

\section{Acute Toxicity Particles in Mice After Oral Administration}

The mice were observed continuously for $1 \mathrm{~h}$ after administration of the plant fraction intermittently for $4 \mathrm{~h}$, over a period of $24 \mathrm{~h}$ and for 14 days for gross behavioral changes and other signs of toxicity manifestations. After the seventh day of the first administration, the same fractionates were given to the same mice but by doubling the doses. Then again, they were observed for any signs of behavioral change, exudates or external lesions for the following 4 hours and for the next 5 days. No immediate signs or lesions were seen. The present results indicate that

Table 5 Zone of Inhibition (in $\mathrm{mm}$ ) of the Different Concentrations of EA Fraction of A. schimperi Leave Against the Tested Bacteria

\begin{tabular}{|c|c|c|c|c|c|c|}
\hline & \multicolumn{6}{|l|}{ EA } \\
\hline & $200 \mathrm{mg} / \mathrm{mL}$ & $100 \mathrm{mg} / \mathrm{mL}$ & $50 \mathrm{mg} / \mathrm{mL}$ & $25 \mathrm{mg} / \mathrm{mL}$ & Vanc & CAF \\
\hline C. freundii(ATCC 43864) & $\begin{array}{l}14.33 \\
\pm 0.333^{\mathrm{a}^{* * * *} \mathrm{~b} * * * c^{*} * * *} \mathrm{~d} * *\end{array}$ & $\begin{array}{l}11.67 \\
\pm 0.333^{3^{* * * *} b^{* * * *} c^{* * * *} e^{* * *}}\end{array}$ & $8.33 \pm 0.333^{\mathrm{a}^{* * * *} \mathrm{~d}^{* * * *} \mathrm{e}^{* * * *}}$ & $\begin{array}{l}6.83 \pm \\
0.167^{\mathrm{a} * * * *} \mathrm{~d}^{* * * *} \mathrm{e} * * *\end{array}$ & $\begin{array}{l}22.33 \\
\pm 0.33\end{array}$ & $\begin{array}{l}22.67 \\
\pm 0.33\end{array}$ \\
\hline $\begin{array}{l}\text { K. pneumonia (ATCC } \\
700603 \text { ) }\end{array}$ & $\begin{array}{l}15.67 \\
\pm 0.667^{7^{* * * *} b^{* * * *} c^{* * * * *}}\end{array}$ & $14 \pm 0.577^{2 * * * \mathrm{~b} * * * c^{* * * *}}$ & $9.67 \pm 0.333^{3^{* * *} \mathrm{~d} * * * \mathrm{e}^{* * * *}}$ & $\begin{array}{l}8.17 \\
\pm 0.167^{7^{* * * *} d^{* * * *} e^{* * * *}}\end{array}$ & $\begin{array}{l}24.00 \\
\pm 0.57\end{array}$ & $\begin{array}{l}21.67 \\
\pm 0.66\end{array}$ \\
\hline S. typhi (ATCC 6539) & $\begin{array}{l}18.25 \pm \\
0.00^{\mathrm{a}^{* * *} \mathrm{~b}^{* * * *} \mathrm{c}^{* * * *} \mathrm{~d} * * *}\end{array}$ & $\begin{array}{l}15.33 \\
\pm 0.333^{\mathrm{a}^{* * * *} \mathrm{~b} * * *} \mathrm{c}^{* * *} \mathrm{e}^{* * *}\end{array}$ & $\begin{array}{l}10.33 \\
\pm 0.00^{\mathrm{a}^{* * * *} \mathrm{~b}^{* * *} \mathrm{~d}^{* * * *} \mathrm{e}^{* * *}}\end{array}$ & $\begin{array}{l}8.18 \\
\pm 0.167^{a^{* * * *} c^{* * *} d^{* * * *} e^{* * * *}}\end{array}$ & $\begin{array}{l}22.67 \\
\pm 0.66\end{array}$ & $\begin{array}{l}22.33 \\
\pm 0.33\end{array}$ \\
\hline E. coli (ATCC 25922)) & $\begin{array}{l}16.67 \\
\pm 0.333^{\mathrm{a}^{* * * *} \mathrm{~b}^{* * * *} \mathrm{c}^{* * * *} \mathrm{~d}^{* *}}\end{array}$ & $\begin{array}{l}14.5 \\
\pm 0.00^{\mathrm{a}^{* * * *} \mathrm{~b} * * * * c^{* * * * *} \mathrm{~d} * *}\end{array}$ & $\begin{array}{l}11.88 \\
\pm 0.333^{\mathrm{a}^{* * *} \mathrm{~b}^{* * * *} \mathrm{~d}^{* * * *} \mathrm{e}^{* * * *}}\end{array}$ & $\begin{array}{l}7.5 \\
\pm 0.289^{\mathrm{a}^{* * * *} \mathrm{c} * * * \mathrm{~d} * * *} \mathrm{e}^{* * * *}\end{array}$ & $\begin{array}{l}24.00 \\
\pm 0.57\end{array}$ & $\begin{array}{r}23.33 \\
\pm 0.33\end{array}$ \\
\hline$P$. aeruginosa & $\begin{array}{l}17.67 \pm \\
0.333^{\mathrm{a}^{* * *+b^{* * * * *}} \mathrm{c}^{* * * * d^{* * *}}}\end{array}$ & $\begin{array}{l}15.33 \pm \\
0.333^{\mathrm{a}^{* * *} \mathrm{~b}^{* * * *} \mathrm{c} * * *} \mathrm{e}^{* *}\end{array}$ & $12 \pm 0.57^{\mathrm{a}^{* * * * * * * * * * * * * *}}$ & $\begin{array}{l}10.67 \\
\pm 0.667^{\mathrm{d}^{* * * *} \mathrm{~d}^{* * * *} \mathrm{e}^{* * * *}}\end{array}$ & $\begin{array}{l}22.33 \\
\pm 0.66\end{array}$ & $\begin{array}{l}22.33 \\
\pm 0.66\end{array}$ \\
\hline S. aureus(ATCC 292I3) & 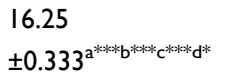 & $15 \pm 0.33^{\mathrm{a}^{* * * *} \mathrm{~b} \mathrm{~b}^{* * * *} \mathrm{c}^{* * *} \mathrm{e}^{*}}$ & $\begin{array}{l}10.33 \\
\pm 0.333^{\mathrm{a}^{* * * *} \mathrm{~b} * * * \mathrm{~d}^{* * * *} \mathrm{e}} \mathrm{e}^{* * * *}\end{array}$ & $\begin{array}{l}7 \\
\pm 0.577^{\mathrm{a}^{* * * *} \mathrm{c}^{* * * *} \mathrm{~d} * * * *} \mathrm{e}^{* * * *}\end{array}$ & $\begin{array}{r}18.33 \\
\pm 0.33\end{array}$ & $\begin{array}{l}22.33 \\
\pm 0.33\end{array}$ \\
\hline
\end{tabular}

Notes: Values are expressed as Mean \pm S.E.M $(n=3)$, analysis was performed with One-Way ANOVA followed by Tukey's test; ${ }^{a}$ Compared to positive control, ${ }^{\text {b}} \mathrm{To} 25 \mathrm{mg} /$ $\mathrm{mL}$, ${ }^{\mathrm{C}} \mathrm{To} 50 \mathrm{mg} / \mathrm{mL}$, ${ }^{\mathrm{d}} \mathrm{To} 100 \mathrm{mg} / \mathrm{mL}$, ${ }^{\mathrm{e}} \mathrm{To} 200 \mathrm{mg} / \mathrm{mL}$; ${ }^{*} \mathrm{P}<0.05$, **P $<0.01, * * * \mathrm{P}<0.001$. The negative control has shown no antibacterial activity.

Abbreviations: CAF, chloramphenicol; Vacho, vancomycin. 


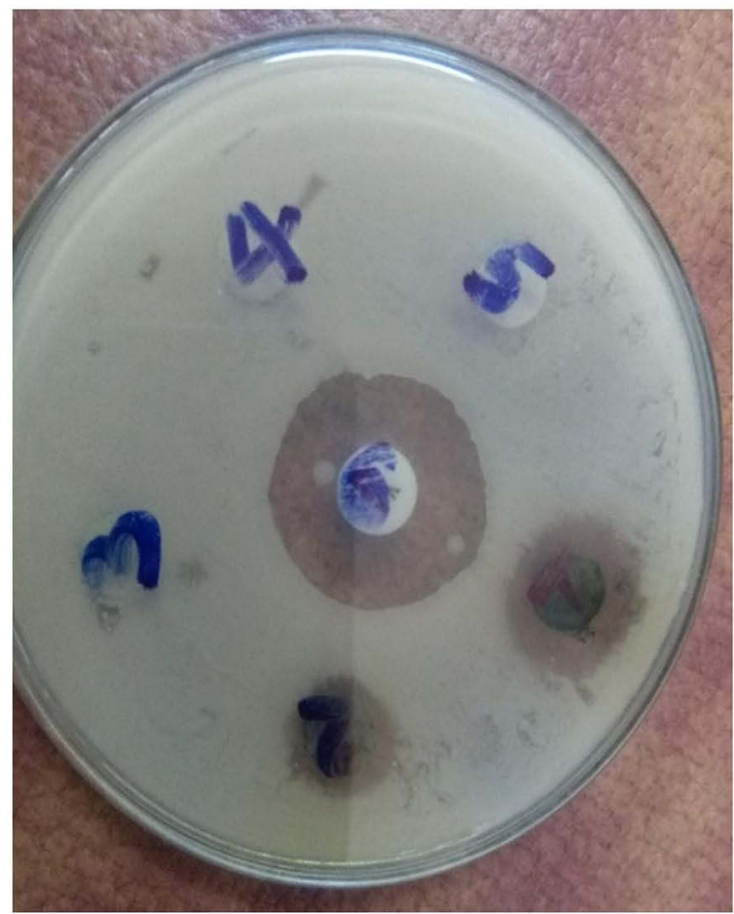

A

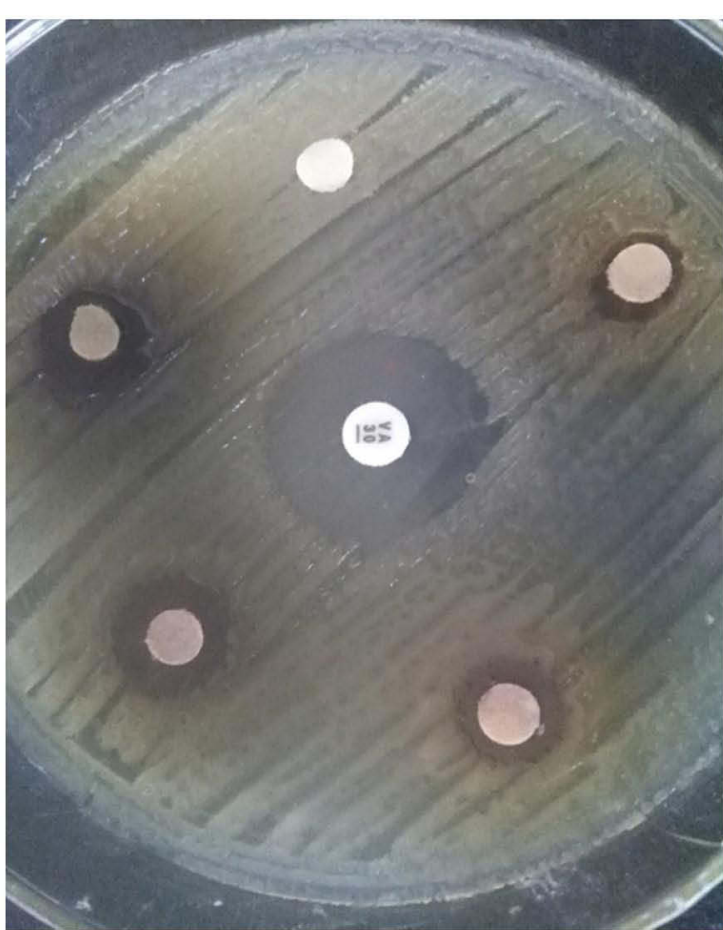

B

Figure I Zone of inhibition of A. schimperi TCM (A) and EA (B) fraction against S. typhi(+ve CAF disc as a positive control and 5\%DMSO as a negative control).

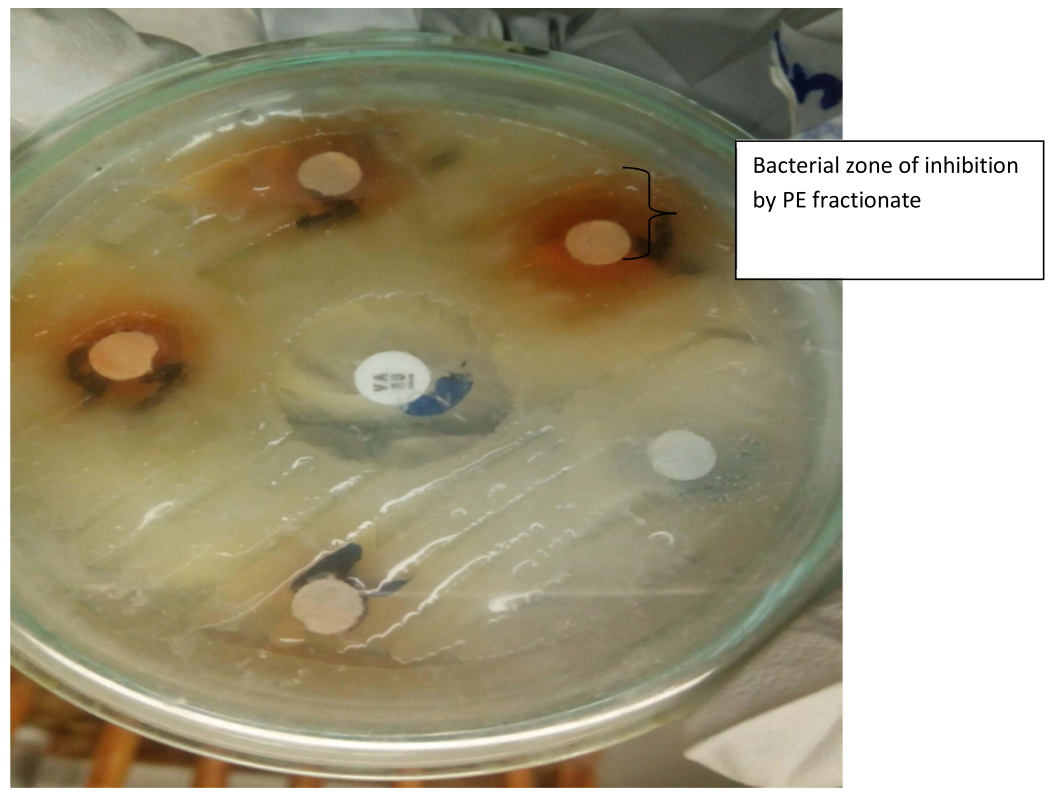

Figure 2 Agar well diffusion test by PE fractionate of the leaves of A. schimperi against K. pneumoniae. (+ve CAF disc as a positive control and $5 \%$ DMSO as a negative control). 
Table 6 The MIC (in $\mathrm{mg} / \mathrm{mL}$ ) of the Solvent Fractions of the Leaves of A. schimperi Against Tested Bacteria

\begin{tabular}{|l|l|l|l|l|}
\hline \multirow{2}{*}{ Bacteria } & \multicolumn{2}{l|}{ DCM Fraction } & \multicolumn{2}{l|}{ PE Fraction } \\
\cline { 2 - 5 } & MIC & MBC & MIC & MBC \\
\hline K. pneumoniae & $4.17 \pm 0.00$ & $6.95 \pm 1.39$ & $11.13 \pm 2.78$ & $11.13 \pm 2.78$ \\
S. aureus & $13.91 \pm 2.78$ & $16.70 \pm 0.00$ & $13.91 \pm 2.78$ & $19.416 \pm 7.34$ \\
C. freundii & $22.24 \pm 5.54$ & $22.24 \pm 5.54$ & $33.34 \pm 0.00$ & $33.34 \pm 0.00$ \\
E. coli & $11.13 \pm 2.78$ & $13.91 \pm 2.78$ & $13.91 \pm 2.78$ & $16.70 . \pm 0.00$ \\
P. aeruginosa & $13.91 \pm 2.78$ & $13.91 \pm 2.78$ & $11.13 \pm 2.78$ & $13.91 \pm 2.78$ \\
S. typhi & $11.13 \pm 2.78$ & $13.91 \pm 2.78$ & $11.13 \pm 2.78$ & $11.13 \pm 2.78$ \\
\hline
\end{tabular}

Note: The values are the average of triplicate tests.

Abbreviation: MIC, minimum inhibitory concentration.

Table 7 The MIC (in $\mathrm{mg} / \mathrm{mL}$ ) of the Solvent Fractions of the Leaves of A. schimperi Against Tested Bacteria

\begin{tabular}{|l|l|l|l|l|}
\hline \multirow{2}{*}{} & \multicolumn{2}{l|}{ TCM Fraction } & \multicolumn{2}{l|}{ EA Fraction } \\
\cline { 2 - 5 } & MIC & MBC & MIC & MBC \\
\hline E. coli & $16.7 \pm 0.000$ & $22.24 \pm 5.54$ & $22.24 \pm 5.54$ & $16.7 \pm 0.000$ \\
S. typhi & $8.35 \pm 0.000$ & $8.35 \pm 0.000$ & $4.17 \pm 0.000$ & $4.17 \pm 0.000$ \\
S. aureus & $27.79 \pm 5.54$ & $27.79 \pm 5.54$ & $27.79 \pm 5.54$ & $33.34 \pm 0.00$ \\
C. freundii & $44.45 \pm I 1.11$ & $55.56 \pm 11.11$ & $44.45 \pm 11.11$ & $55.56 \pm 11.11$ \\
K. pneumoniae & $27.79 \pm 5.00$ & $33.34 \pm 0.00$ & $33.34 \pm 0.000$ & $33.34 \pm 0.000$ \\
P. aeruginosa & $11.14 \pm 2.79$ & $11.14 \pm 2.79$ & $8.35 \pm 0.000$ & $11.14 \pm 2.79$ \\
\hline
\end{tabular}

Note: The values are the average of triplicate tests.

Abbreviation: MIC, minimum inhibitory concentration.

the leave fractions of $A$. schimperi showed no sign of abnormality at $2000 \mathrm{mg} / \mathrm{kg}$.

\section{Disscusion}

The present study was conducted with the objective of assessing the biologically active secondary metabolites of the plant $A$. schimperi and determining which fractions of the leaf are responsible for its antimicrobial effect. According to the present study, the zone of inhibition of the EA and TCM fractions showed a greater zone of inhibition than that of the DCM and PE fractions at given concentrations against the growth of each test bacterium.

A study conducted in Tanzania on A. schimperi leaf acetone extract inhibited the growth of Salmonella enteritidis, Bacillus cereus, Klebsiella pneumoniae, Escherichia coli, Enterococcus faecalis, Streptococcus thermophilus and Lactobacillus bulgaricus with an MIC value ranging between 0.7812 and $25 \mathrm{mg} / \mathrm{mL}$ against microbes tested in this study. ${ }^{29}$ Likewise, a study conducted in Ethiopia on A. schimperi methanolic and water extracts showed the growth inhibition of $S$. aureus, $S$. pyogenes, E. coli, P. aeruginosa and $P$. vulgaris. ${ }^{14}$ Citrobacter freundii appears to be the least susceptible bacteria for all fractions of the plant, with the respective mean zone of inhibition at the highest concentration $(200 \mathrm{mg} / \mathrm{mL})$.

Phytochemical compounds commonly associated with combating microbial resistance and having antimicrobial activity in medicinal plants are flavonoids, alkaloids, tannins, terpenoids, essential oils, saponins, and phenols. Even though, at this point in time, it is difficult to judge the mechanism of action of the bioactivity of the solvent fractions of the study plant, it is plausible to speculate about their antibacterial effect based on the different mode of action of the bioactive secondary metabolites detected in the phytochemical analysis of this study. ${ }^{30}$

The possible mechanism of antibacterial activities of alkaloid compounds might be disruption of cell membranes or inhibition of protein synthesis of bacteria. Phenols affect bacterial metabolism, possibly by precipitating of proteins. ${ }^{25}$ The antimicrobial activity of saponins on microorganisms is due to their interaction with the sterol moiety, which is present in the membrane of microbes and causes destruction of microbial cell membranes, causing leakage of cell contents. ${ }^{31}$ On the other hand, flavonoids inhibit a perplexing number and variety 
of prokaryotic enzymes and methylation of DNA is another way of antimicrobial activity of flavonoids. ${ }^{32}$

According to the present study, the result of the antibacterial activity test indicated that both the solvent fractions were found to have greater antibacterial effects against all the test bacteria with varying degrees of antibacterial activity spectrum. The reason could be due to the differences in the composition and concentrations of the secondary metabolites in the fractions. Staphylococcus aureus was the least susceptible bacteria to the chloroform and ethyl acetate fraction at equal concentrations compared to the other tested bacteria. ${ }^{33}$

Furthermore, the differences in the antibacterial spectrum of the ethyl acetate and the chloroform fraction against the test bacteria might be linked to the differences in the composition and the concentration of the secondary metabolites in the respective fractions. ${ }^{31}$

The groups of phytochemical compounds commonly associated with combating microbial resistance and having antimicrobial activity in medicinal plants are flavonoids, alkaloids, tannins, tritepenoids, essential oils, saponins, glycosides, and phenols. ${ }^{34}$

The presence of flavonoids in ethyl acetate and chloroform faction of the leaf of $A$. schimperi could contribute their own share for the observed antibacterial activities. The possible mechanism of action for the antibacterial effects of flavonoids includes damage or disruption of the cell membranes and inhibition of the synthesis of nucleic acids, which can lead to the death of the susceptible bacterium. ${ }^{35}$

Phenols are the other phytochemicals that might be involved in the antibacterial activities of medicinal plants used for different infectious diseases. The extracts of phenolic compounds have been found to have a growth inhibition effect on different bacteria (E. coli, S. aureus and Salmonella typhi). ${ }^{36}$

Tannins are the other compounds that have been found to have antimicrobial activities against the growth of bacteria. The antibacterial role of tannin constituents has been shown to inhibit and decrease the load and growth of aerobic, mouth cavity colonizing bacteria. ${ }^{37}$

According to the qualitative test of this study anthraquinones have been detected in the fraction of the leaves of A. schimperi. Thus, the antibacterial activities of the ethyl acetate and the chloroform fractions of the present study might be related to the presence of anthraquinones. This antibacterial action of anthraquinones has been strengthened by the growth inhibitory effect of different anthraquinone compounds such as emodin (isolated from Rheum officinale). ${ }^{38}$

The plausible antibacterial activity of anthraquinones has been associated with their interaction with the bacterial cell wall and cell membrane components, which can lead to the death of the bacteria as a result of leakage of cytoplasmic components and loss of cell integrity. ${ }^{39}$

\section{Conclusion and Recommendations}

Acokanthera schimperi is one of medically important plants which have variety of effects on treating infectious diseases. The present study revealed that the solvent fractions of the leaves of $A$. schimperi have antibacterial activities against the growth of the selected bacterial species with varying antibacterial spectrum. Therefore, the study provides a scientific basis for the traditional claimed use of the medicinal plant for the treatment of bacterial infections.

\section{Acknowledgment}

The authors thank the Ethiopian Public Health Institute (EPHI) and the National Animal Health Diagnostic and Investigation Center (NAHDIC) for their kind gift of bacterial strains.

\section{Funding}

The authors thank the University of Gondar, Research and Community Service Vice President Office for its megaprogram.

\section{Disclosure}

The authors report no conflicts of interest in this work.

\section{References}

1. Kalayou S, Haileselassie M, Gebre-egziabher G, et al. In-vitro antimicrobial activity screening of some ethnoveterinary medicinal plants traditionally used against mastitis, wound and gastrointestinal tract complication in Tigray Region, Ethiopia. Asian Pac J Trop Biomed. 2012;2(7):516-522. doi:10.1016/S2221-1691(12)60088-4

2. Girmay T, Teshome Z. Assessment of traditional medicinal plants used to treat human and livestock ailments and their threatening factors in Gulomekeda District, Northern Ethiopia. Int J Emerg Trends Sci Technol. 2017;4(4):5061-5070. doi:10.18535/ijetst/v4i4.03

3. Avigdor E, Wohlmuth H, Asfaw Z, Awas T. The current status of knowledge of herbal medicine and medicinal plants in fiche, Ethiopia. J Ethnobiol Ethnomed. 2014;10(1):1-33.

4. Tilahun T, Giday M. Ethnobotanical study of medicinal plants used by people in Zegie peninsula, Northwestern Ethiopia. J Ethnobiol Ethnomed. 2007;3(1):1.

5. Araya S, Abera B, Giday M. Study of plants traditionally used in public and animal health management in Seharti Samre District, Southern Tigray, Ethiopia. J Ethnobiol Ethnomed. 2015;11(1):22. doi:10.1186/s13002-015-0015-5 
6. Chekole G, Asfaw Z, Kelbessa E. Ethnobotanical study of medicinal plants in the environs of tara-gedam and amba remnant forests of libo kemkem district, Northwest Ethiopia. J Ethnobiol Ethnomed. 2015;11 (1):1-38.

7. Belayneh A, Zemede A, Sebsebe D, Bussa. NF. Medicinal plants potential and use by pastoral and agro-pastoral communities in erer valley of babile wereda, eastern Ethiopia. J Ethnobiol Ethnomed. 2012;8(1):1. doi:10.1186/1746-4269-8-42

8. Belayneh A, Bussa NF. Ethnomedicinal plants used to treat human ailments in the prehistoric place of harla and dengego valleys, Eastern Ethiopia. J Ethnobiol Ethnomed. 2014;10(1):1-17.

9. Teklay Abraha BA, Giday M. An ethnobotanical study of medicinal plants used in kilte awulaelo district, Tigray region. J Ethnobiol Ethnomed. 2013;9(1):1-23.

10. Nanyingi MO, Nirb DM, Zante GZ. Ethnopharmacological survey of Samburu district, Kenya. J Ethnobiol Ethnomed. 2008;12(12). Available from: http://www.ethnobiomed.com/content/4/1/14. Accessed August 24, 2021.

11. Mohammed T, Erko B, Giday M. Evaluation of antimalarial activity of leaves of acokanthera schimperi and croton macrostachyus against Plasmodium berghei in swiss albino mice. BMC Complement Altern Med. 2014;14(1):314.

12. Chaurasia S, Sharma P. Evaluation of antibacterial and antimutagenic potential of acokanthera oppositifolia and leucaena leucocephala. Am J Pharm Health Res. 2015;3:246-258.

13. Mabona U, Viljoen A, Shikanga E, Marston A, Van Vuuren S. Antimicrobial activity of southern African medicinal plants with dermatological relevance: from an ethnopharmacological screening approach, to combination studies and the isolation of a bioactive compound. J Ethnopharmacol. 2013;148(1):45-55. doi:10.1016/j. jep.2013.03.056

14. Taye B, Giday M, Animut A, Seid J. Antibacterial activities of selected medicinal plants in traditional treatment of human wounds in Ethiopia. Asian Pac J Trop Biomed. 2011;1(5):370-375. doi:10.1016/S2221-1691(11)60082-8

15. Tadeg H, Mohammed E, Asres K, Gebre-Mariam T. Antimicrobial activities of some selected traditional Ethiopian medicinal plants used in the treatment of skin disorders. J Ethnopharmacol. 2005;100(12):168-175. doi:10.1016/j.jep.2005.02.031

16. Grace D, Randolph $\mathrm{T}$, Affognon $\mathrm{H}$, Dramane D, Diall O, Clausen P-H. Characterisation and validation of farmers' knowledge and practice of cattle trypanosomosis management in the cotton zone of West Africa. Acta Trop. 2009;111(2):137-143. doi:10.1016/j. actatropica.2009.03.009

17. Landers T, Cohen B, Wittum TE, Larson EL. A review of antibiotic use in food animals: perspective, policy, and potential. Public Health Rep. 2012;127(1):4-22. doi:10.1177/003335491212700103

18. Hossain MA, Al-Toubi WAS, Weli AM, Al-Riyami QA, Al-Sabahi $\mathrm{JN}$. Identification and characterization of chemical compounds in different crude extracts from leaves of Omani neem. J Taibah Univ Sci. 2013;7(4):181-188. doi:10.1016/j.jtusci.2013.05.003

19. Ayoola G, Coker H, Adesegun S, et al. Phytochemical screening and antioxidant activities of some selected medicinal plants used for malaria therapy in Southwestern Nigeria. Trop J Pharm Res. 2008;7 (3):1019-1024.

20. Farhan H, Malli F, Rammal H, et al. Phytochemical screening and antioxidant activity of Lebanese Eryngium creticum L. Asian Pac $J$ Trop Biomed. 2012;2(3):S1217-S1220. doi:10.1016/S22211691(12)60388-8

21. Mansouri S, Foroumadi A, Ghaneie T, Najar AG. Antibacterial activity of the crude extracts and fractionated constituents of myrtus communis. Pharm Biol. 2001;39(5):399-401. doi:10.1076/ phbi.39.5.399.5889
22. Arunkumar S, Muthuselvam M. Analysis of phytochemical constituents and antimicrobial activities of Aloe vera L. against clinical pathogens. World J Agric Sci. 2009;5(5):572-576.

23. Demeke Debalke MB, Kenubih A, Yayeh M. Assessment of antimicrobial effects of aqueous-ethanolic extract of sida rhombofolia's aerial part. Sci World J. 2018;2018: 8429809. doi:10.1155/2018/ 8429809

24. CLSI. Clinical Microbiology Procedures Handbook. Vol. 3. Washington, DC, USA: ASM Press; 2016.

25. Molla Y, Nedi T, Tadesse G, Alemayehu H, Shibeshi W. Evaluation of the in vitro antibacterial activity of the solvent fractions of the leaves of Rhamnus prinoides l'herit (Rhamnaceae) against pathogenic bacteria. BMC Complement Altern Med. 2016;16(1):278. doi:10.1186/s12906-016-1279-6

26. Belay D, Kenubih A, Yesuf M, Kebede E, Yayeh M, Birhan M. Antioxidant and antimicrobial activity of solvent fractions of Calpurnia aurea (Ait.) Benth. (Fabaceae). J Exp Pharmacol. 2021;13:499-509. doi:10.2147/JEP.S285872

27. Parekh A, Truong MT, Pashtan I, et al. Acute gastrointestinal toxicity and tumor response with preoperative intensity modulated radiation therapy for rectal cancer. Gastrointest Cancer Res. 2013;6(5-6):137.

28. OECD. Ageing and Transport: Mobility Needs and Safety Issues. Organization for Economic; 2001.

29. Makirita WE, Chauka LJ, Chacha M. Antimicrobial and cytotoxicity activity of Clausena anisata, acokanthera shemperii and Olea europaea growing in Tanzania. Eur J Med Plants. 2016;14(2):2. doi:10.9734/EJMP/2016/23635

30. Cowan MM. Plant products as antimicrobial agents. Clin Microbiol Rev. 1999;12(4):564-582.

31. Mattana C, Satorres S, Sosa A, Fusco M, Alcaráz L. Antibacterial activity of extracts of acacia aroma against methicillin-resistant and methicillin-sensitive Staphylococcus. Braz J Microbiol. 2010;41 (3):581-587. doi:10.1590/S1517-83822010000300007

32. Cushnie TPT, Lamb AJ. Antimicrobial activity of flavonoids. Int $J$ Antimicrob Agents. 2005;26(5):343-356. doi:10.1016/j. ijantimicag.2005.09.002

33. Pantosti A, Sanchini A, Monaco M. Mechanisms of antibiotic resistance in Staphylococcus aureus. Future Microbiol. 2007;2 (3):323-334. doi:10.2217/17460913.2.3.323

34. Neog P, Choudhury S, Bhattacharjee A, Chetia P, Choudhury MD. A review on ethnomedicinal plants and their active secondary metabolites in antibacterial drug discovery research. Pleione. 2013;7:413-423.

35. Dzoyem JPHH, Ngameni B, Ngadjui BT, Sekimizu K. Antimicrobial activity of flavonoids. Int J Antimicrob Agents. 2013;26:343-356.

36. Thippeswamy NB, Naidu KA, Achur RN. Antioxidant and antibacterial properties of phenolic extract from Carum carvi L. J Pharm Res. 2013;7:352-357.

37. Moghbel A, Farjzadeh A, Aghel N, Agheli H, Rais N. The effect of green tea on prevention of mouth bacterial infection, halitosis, and plaque formation on teeth. Iran J Toxicol. 2011;5:502-515.

38. Liu B, Xie J, Ge X, et al. Comparison study of the effects of anthraquinone extract and emodin from Rheum officinale bail on the physiological response, disease resistance of Megalobrama amblycephala under high temperature stress. Turk J Fish Aquat Sci. 2012;12:905-916.

39. Wei Y, Liu Q, Yu J, et al. Antibacterial mode of action of 1,8-dihydroxy-anthraquinone from porphyra haitanensis against Staphylococcus aureus. Nat Prod Res. 2014;29:976-979. doi:10.1080/14786419.2014.964705 
Journal of Experimental Pharmacology

Dovepress

\section{Publish your work in this journal}

The Journal of Experimental Pharmacology is an international, peerreviewed, open access journal publishing original research, reports, reviews and commentaries on all areas of laboratory and experimental pharmacology. The manuscript management system is completely

online and includes a very quick and fair peer-review system. Visit http://www.dovepress.com/testimonials.php to read real quotes from published authors.

Submit your manuscript here: https://www.dovepress.com/journal-of-experimental-pharmacology-journal 